\title{
Canadian Public Health Laboratory Network: Prioritized support for northern, remote and isolated communities in Canada
}

\author{
Respiratory Virus Infections Working Group ${ }^{1}$
} \author{
2020;46(10):322-3. https://doi.org/10.14745/ccdr.v46i10a02 \\ Keywords: COVID-19, Canada, point-of-care testing
}

Suggested citation: Respiratory Virus Infections Working Group. Canadian Public Health Laboratory Network: Prioritized support for northern, remote and isolated communities in Canada. Can Commun Dis Rep

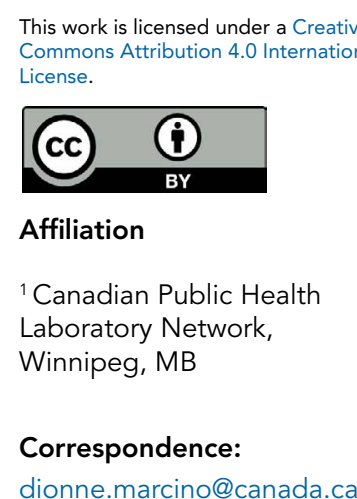

dionne.marcino@canada.ca
In the spirit of Truth and Reconciliation, and in an effort to ensure equitable healthcare access, the Canadian Public Health Laboratory Network (CPHLN) advocates for the prioritized distribution of point-of-care supplies to northern, remote and isolated (NRI) communities. These communities have significantly greater proportions of First Nations, Metis and Inuit peoples than communities in southern Canada and often experience obstacles related to rapid access to conventional health care, such as diagnostic testing services, and to linkage to care. On-site services may not be available and challenges with specimen transport can lead to increased turnaround time and delays in diagnosis/treatment for affected individuals. These delays may then lead to further challenges in contact tracing and implementation of effective public health measures to contain transmission networks.

There are only a limited number of near-care or point-of-care (POC) testing options currently approved in Canada for detecting severe acute respiratory syndrome coronavirus 2 (SARS-CoV-2), the virus that causes coronavirus disease 2019 (COVID-19). Testing equipment and supplies are severely limited, leading to delays in testing. Current data demonstrate that most transmissions occur in the first five days of illness and, as such, delays in diagnostic turnaround times maximize the potential for spread of SARS-CoV-2. As a result, it is critical to prioritize the distribution of SARS-CoV-2 POC testing supplies to areas where they will have the greatest public health impact, including NRI communities throughout Canada.

The only option for SARS-CoV-2 POC testing currently available in Canada for use outside a laboratory is the Xpert ${ }^{\circledR}$ Xpress SARS-CoV-2 assay, which operates on the GeneXpert ${ }^{\circledR}$ system (Cepheid, Sunnyvale California, United States). The Xpert Xpress SARS-CoV-2 assay has a rapid turnaround time (approximately 50 minutes) with an option for Early Assay Termination as early as 30 minutes for positive specimens. As the GeneXpert system is used to test for many common infections, more than 200 instruments are already distributed across Canada, primarily in urban centres in the south. Urban centres also have access to centralized, laboratory-based testing, with high-throughput commercial or laboratory-developed tests.

Centralized laboratory-based testing is unavailable or difficult to access for NRI communities. To date, 61 POC devices and more than 9,100 tests have been distributed to remote communities and current allocations of tests remain limited. As such, it is essential to develop a coordinated approach for test distribution to ensure that NRI communities receive appropriate supplies to provide adequate diagnostic support.

Because they do not have timely access to centralized laboratory-based testing, some NRI communities have turnaround times for POC SARS-CoV-2 testing that can extend beyond 7-10 days. In addition, risk factors for severe COVID-19 cases are prevalent within NRI communities, including diabetes and heart disease. Thus, there is concern that the spread of COVID-19 within and between these communities will stress the existing healthcare system. The allocation of Canada's limited resources should be guided by ethical principles. In this case, equitable and fair distribution of resources in accordance to the principle of justice is paramount in addressing the needs, vulnerabilities and consequent health inequities experienced by those living in NRI communities.

It is the position of CPHLN that specific, dedicated support should be provided for NRI communities. Provincial and territorial health authorities that have access to conventional, laboratory-based diagnostic testing options are encouraged 
to prioritize near-care/POC equipment and testing supplies to NRI communities. While recognizing that each jurisdiction is unique, CPHLN recommends that provinces and territories develop a coordinated approach to deploy a significant proportion of their Xpert Xpress SARS-CoV-2 assay supply to NRI communities.

\section{Key points from this statement}

- $\quad$ The Xpert Xpress SARS-CoV-2 assay POC testing supplies are critically low across Canada.

- The majority of these POC testing supplies are being utilized in urban centres where other testing options are available.

- Because of these supply shortages, it can take up to 7-10 days to receive a SARS-CoV-2 test result in NRI communities.

- Diseases that are associated with severe COVID-19 infection, such as diabetes and heart disease, are prevalent in NRI communities.
- $\quad$ The POC testing supplies should be prioritized for the vulnerable NRI communities who lack equitable access to conventional testing services and where they will have the greatest impact on public health.

- Starting immediately, until there is an increase in the allocation of supplies to Canada, health authorities should consider redeploying a significant proportion of Xpert Xpress SARS-CoV-2 assay testing supplies to NRI communities to support the current and future pandemic waves.

- This prioritization of POC SARS-CoV-2 testing is in respect of the principle of justice, thereby ensuring equitable distribution of resources according to need and promoting well-being for those who otherwise lack equivalent options for rapid diagnostic services.

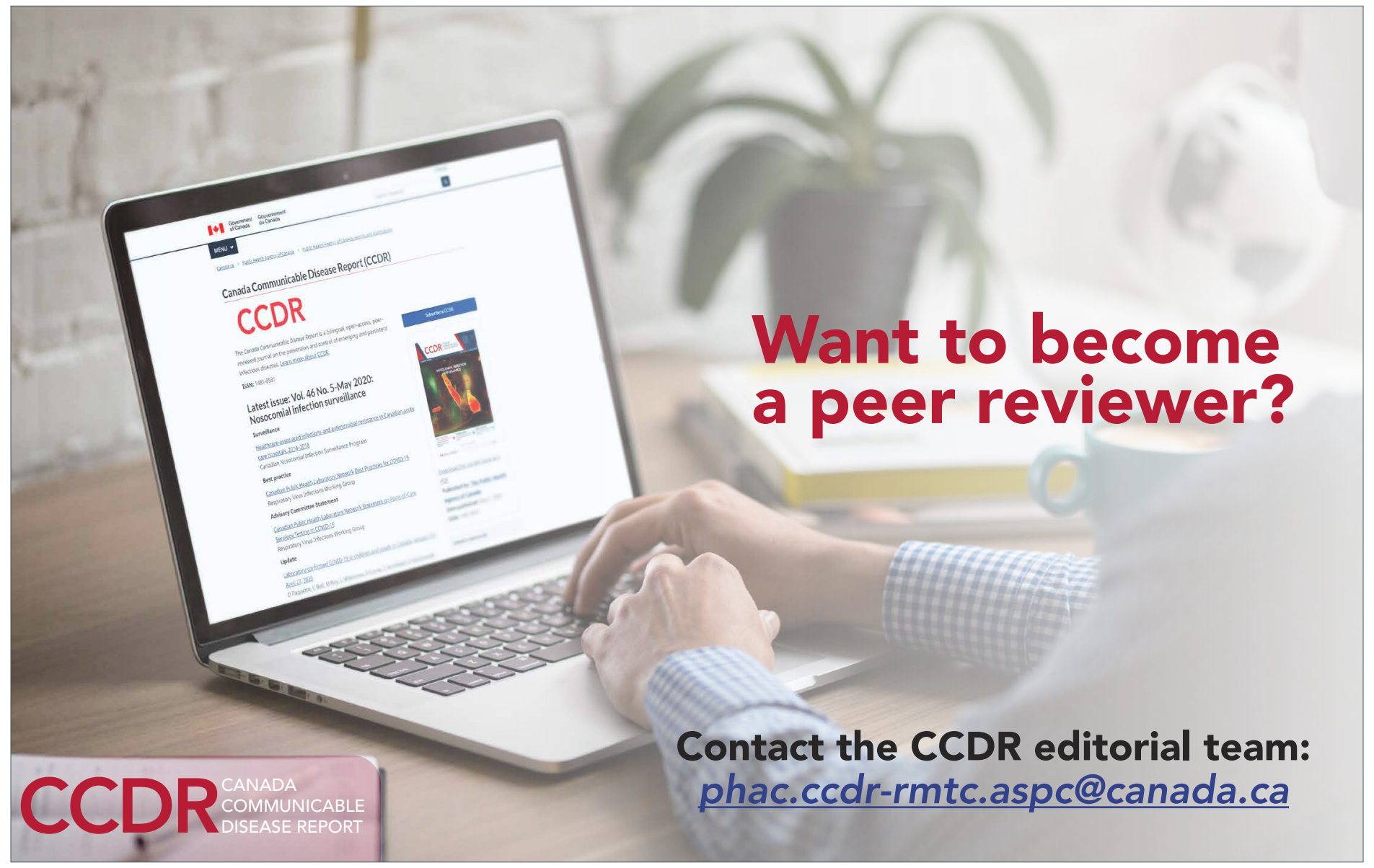

